\title{
PROJETO LARGA ESCALA: UMA ANÁLISE A PARTIR DA BIBLIOGRAFIA EXISTENTE
}

\section{Project on a large scale: an analysis according to the existing bibliography}

\section{Proyecto escala larga: un análisis de la bibliografia existente}

Greicelene Aparecida Hespanhol Bassinello ${ }^{1}$

Maria Helena Salgado Bagnato ${ }^{2}$

\section{RESUMO}

Este trabalho consistiu em mapear e discutir a produção acadêmica referente ao Projeto Larga Escala. Ele se insere em um projeto mais abrangente que objetivou reconstituir e compreender a história desta experiência pedagógica. Adotamos, para o levantamento bibliográfico, bases de dados que permitiam acesso a teses, dissertações e periódicos. Os resultados foram apresentados de forma descritiva, segundo um conjunto que engloba a produção existente. Podemos ver, com essa primeira aproximação, o pequeno número de investigações realizadas e o desconhecimento dos resultados do Projeto Larga Escala em âmbitos locais e regionais. Desta forma, este estudo nos permitiu verificar que o projeto foi pouco investigado, considerando que seu desenvolvimento ocorreu em todo o país durante um período de tempo relativamente longo.

Palavras-chave: História da Enfermagem. Educação Profissionalizante. Pesquisa em Educação de Enfermagem.

\begin{abstract}
This work consisted of discussing the academic production referring to the Project on a Large Scale - PLS. It is part of a bigger project which motivated the rebuilding and comprehension of the history of this pedagogical experience. We adopted, for bibliography, data basis that allowed access to thesis, dissertations and periodicals. The results were presented in a descriptive way, according to a set containing the existing production. It is possible to see, with this first approach, the small number of investigations done and the lack of knowledge on the PLS results on a local or regional scale. According to this, the study allowed us to conclude that the project was little investigated, considering that it was developed through all the country in a relatively long period.
\end{abstract}

\section{Resumen}

Este trabajo consistió en mapear y discutir la producción académica con respecto a Proyecto Escala Larga. El está inserido en un proyecto más completo que planeó reconstruir y entender la historia de esta experiencia pedagógica. Adoptamos para el torno bibliográfico, bases de los hechos que permitió el acceso a teorías, disertaciones y periódicos. Los resultados fueron presentados de forma descriptiva, según un conjunto que engloba la producción existente. Podemos ver, con que se acerca primero, el pequeño número de investigaciones realizadas y la falta de familiaridad de los resultados del Proyecto Escala Larga en alcances regionales y locales. De esta manera, este estudio nos permitió verificar que el proyecto fue poco investigado, acerca de que su desarrollo ocurrió en todo el país durante un período de tiempo relativamente largo.
Keywords: History of nursing. Professional education. Nursing education research.
Palabras clave: Historia de la Enfermería. Educación profesional. Investigación en educación de enfermería

${ }^{1}$ Enfermeira. Doutora em Educação. Docente das Faculdades Integradas Einstein de Limeira - FIEL/Limeira. Enfermeira da Secretaria de Estado da Saúde de São Paulo - DRS X - Piracicaba. Membro do Laboratório de Práticas de Educação e Saúde - PRAESA/FE/UNICAMP. Brasil. E-mail: jlabas@terra.com.br, Enfermeira. Livre-docente da Faculdade de Educação da Universidade Estadual de Campinas - UNICAMP. Pós-Doutorado em Educação pela Universidade de Barcelona. Coordenadora do Laboratório de Práticas de Educação e Saúde - PRAESA/FE/UNICAMP. Brasil. E-mail: mbagnato@unicamp.br 


\section{INTRODUCÃO}

0 interesse pela educação profissional em nível médio de Enfermagem, seus aspectos históricos, a formação de alunos neste nível e os desafios a ela relacionados nos mobilizou para o desenvolvimento deste estudo. Persistem, neste 'lócus' de formação, significantes lacunas e pouca produção de trabalhos, o que dificulta a socialização e a avaliação de programas e projetos, bem como seu impacto na prática.

Os projetos de formação profissional em nível médio de Enfermagem, como o Projeto Larga Escala, têm se apresentado de forma descontínua, por diversos fatores - nem todos com muita visibilidade - tais como o não-alcance das metas propostas, seu custo demasiado alto, a perda de apoio político e as implicações das reformas administrativas desencadeadas no início de um novo governo.

Com alguns destes questionamentos e visando contribuir para dar visibilidade a aspectos deste Projeto, este texto foi construído com a intenção de fazer uma revisão das produções existentes sobre o mesmo (através de artigos, documentos e publicações disponíveis). Esta preocupação se inseriu em um projeto de pesquisa de doutorado mais abrangente que objetivou reconstituir e compreender a história do Projeto de Formação de Trabalhadores para a Área de Saúde em Larga Escala, também conhecido como Projeto Larga Escala - PLE, desenvolvido no período de fevereiro de 2004 a março de 2007

Para Santos ${ }^{1}$, a participação de enfermeiros em pesquisas é essencial para edificar o saber e a produção de conhecimento e deve ser um meio para revelar e refletir sobre a história, o ensino, a prática e a inserção da enfermagem na sociedade questões relevantes para posterior compreensão de aspectos relacionados ao desenvolvimento da Enfermagem Brasileira.

Assim, buscamos mapear e dialogar com o que foi produzido sobre o PLE e explicitar aspectos ainda pouco explorados dessa história.

\section{SITUANDO O PROJETO LARGA ESCALA}

0 Projeto de Formação de Trabalhadores para a Área de Saúde em Larga Escala, também conhecido como Projeto Larga Escala - PLE, surgiu em 1981, fruto do processo de reformulação dos serviços de saúde que visavam à extensão de cobertura - a implantação acelerada de uma rede básica de unidades de saúde, com prioridade para as populações rurais de pequenos centros e da periferia das grandes cidades.

As unidades federadas da região nordeste, dentre elas 0 Piauí, Alagoas e Rio Grande do Norte, foram os primeiros a adotar a experiência pedagógica, inicialmente voltada à formação dos visitadores sanitários, preparados pelas próprias instituições de saúde, visto que tal profissional, como parte integrante da equipe de enfermagem, era legitimado e reconhecido pela sociedade, atuante na área de saúde pública.

O PLE foi sendo construído no decorrer da sua implantação nos Estados, e o documento inicial datado de 1982, denominado
Recursos Humanos para Serviços Básicos de Saúde: Formação de Pessoal de Níveis Médio e Elementar pelas Instituições de Saúde ${ }^{1}$, sistematiza a proposta do Larga Escala e indica certas estratégias que, de certa forma, viriam superar os processos de formação anteriores, como os mecanismos de legitimação desta capacitação por intermédio da cooperação entre Ministérios da Saúde e Educação, promoção profissional e intelectual dos trabalhadores, metodologia adequada à realidade dos serviços, compreendendo que estes profissionais de nível médio e elementar possuíam certa autonomia de trabalho em locais que, pela suas condições socioeconômicas, não dispunham de médicos ou outros profissionais de nível superior $^{2}$.

A construção desta experiência pedagógica foi firmada a partir de algumas possibilidades aventadas pelo Capítulo IV da Lei das Diretrizes e Bases da Educação Nacional - LDB n. ${ }^{\circ}$ 5.692, de 11 de agosto de 1971, e pelo parecer do Conselho Federal de Educação - CFE nº 699/72. 0 Capítulo IV refere-se à constituição do ensino supletivo que apresentava a seguinte finalidade: "suprir a escolarização regular para os adolescentes e adultos que não a tenham seguido ou concluído na idade própria; proporcionar, mediante repetida volta à escola, estudos de aperfeiçoamento ou atualização seguido o ensino regular no todo ou em parte" 3:7.

No Brasil, a formação de pessoal de nível médio e elementar é de responsabilidade do sistema de educação, representado na época da implantação do PLE, em nível federal, pelo Ministério da Educação e Conselho Federal de Educação e, nos Estados, pela Secretarias de Estado de Educação e Conselhos Estaduais de Educação. Estruturou-se, portanto, uma instituição educacional específica para o setor saúde, de caráter permanente, articulada ao sistema educacional: os Centros Formadores ${ }^{4}$.

Deste ponto de vista, optou-se por trabalhar uma experiência inovadora, assumindo a educação como prática transformadora e a indicação de um caminho para esta profissionalização. A primeira estratégia do PLE se daria então a partir de situações reais e cotidianas, vividas ou experimentadas pelos próprios trabalhadores no seu ambiente de trabalho, possibilitando 0 surgimento de um indivíduo ativo, produtor de conhecimentos e capaz de modificar o seu meio.

Nesse cenário, foi concebido um projeto comprometido com a transformação social, com uma proposta alternativa de qualificação profissional de trabalhadores sem formação específica, que perdurou até a regulamentação da atual Lei de Diretrizes e Bases da Educação Nacional - LDB, que, em 1996, põe fim aos cursos supletivos, entre eles o PLE.

\section{PRESSUPOSTOS METODOLÓGICOS}

Para este texto, nossa tarefa consistiu em mapear e discutir a produção acadêmica referente ao PLE, por meio dos estudos produzidos sobre o tema.

Os resultados são apresentados de forma descritiva envolvendo a produção existente e divididos em teses e dissertações, artigos científicos e boletins informativos.

Adotamos como fonte de referência para o levantamento bibliográfico bases de dados que permitiam acesso a teses, 
dissertações e periódicos. Nas bibliotecas das universidades, utilizamos o Portal do Conselho de Reitores das Universidades Estaduais Paulistas - CRUESP, que reúne informações sobre os sistemas de Bibliotecas da Universidade de São Paulo - USP, Universidade Estadual Paulista - UNESP e Universidade Estadual de Campinas - UNICAMP, o banco de dados da Universidade Federal do Rio de Janeiro, o sítio do GT de Bibliotecas Virtuais do Instituto Brasileiro de Ciência e Tecnologia, Bibliotecas Acadêmicas do Prossiga Brasil, Biblioteca Virtual de Saúde, banco de teses da Coordenação de Aperfeiçoamento de Nível Superior-CAPES, Associação Brasileira de Enfermagem e BIREME.

Este primeiro momento permitiu visualizar diferentes perspectivas e pluralidade de enfoques dos trabalhos publicados, verificando possíveis lacunas existentes acerca dessa experiência e contribuindo para a originalidade do trabalho.

Segundo Ferreira ${ }^{5}$, o pesquisador tem dois momentos bastante distintos: no primeiro, ele interage com a produção acadêmica, através da quantificação e identificação dos dados; no segundo, ele se pergunta sobre a possibilidade de inventariar esta produção, presumindo tendências, ênfases, escolhas metodológicas e teóricas, aproximando ou diferenciando os trabalhos entre si na escrita da história.

Nossa pesquisa perpassou por esses dois momentos, indispensáveis diante da possibilidade de reconstruir uma história, rastreando aspectos do Larga Escala, como eles se relacionam entre si, quais histórias se assemelham, quais se diferenciam, permitindo a construção de uma plataforma para nossa investigação.

Como primeira tarefa, catalogamos e identificamos o conjunto de teses e dissertações, artigos e boletins informativos; a seguir, ordenamos periodicamente o conjunto de informações e os resultados já obtidos. Para a busca de trabalhos, utilizamos as palavras-chave: Projeto Larga Escala, Ensino Profissionalizante em Enfermagem, Educação Profissional, Qualificação Profissional, Atendentes e Auxiliares de Enfermagem.

0 mapeamento da produção acadêmica se deu pelo acesso às pesquisas por meio dos resumos e trabalhos na íntegra. $\mathrm{Na}$ leitura e análise do material, nos baseamos no objetivo principal da investigação, no procedimento utilizado na abordagem do problema, no tratamento dos dados, no referencial teórico e nos resultados, que nos possibilitaram as conclusões e as recomendações finais.

\section{APRESENTAÇÃO E ANÁLISES DOS RESULTADOS}

$\checkmark$ Conjunto de teses e dissertações

Nesta primeira aproximação com o que foi produzido acerca do Projeto Larga Escala, obtivemos sete dissertações de mestrado, apresentadas no período de 1992 a 2001.
Observamos a distribuição dos estudos por instituição de ensino e ano da defesa, que se configuraram como estudos locorregionais nos Estados do Rio de Janeiro, São Paulo e Bahia, apesar de o PLE existir em âmbito nacional.

Assim, três estudos foram produzidos pela Universidade de São Paulo, as defesas ocorreram nos ano de 1996, 2000 e 2001, respectivamente. A Universidade Federal do Rio de Janeiro contou com a produção de dois trabalhos realizados nos anos de 1992 e 1994.

Já a Universidade Estadual do Rio de Janeiro apresentou uma pesquisa no ano de 1992, seguida pela Universidade Federal da Bahia com o desenvolvimento de um estudo no ano de 1999. Os autores são pesquisadores universitários ligados à área de saúde e educação. Três das sete dissertações foram desenvolvidas em Faculdades de Educação, três em Faculdades de Saúde Pública e uma na Faculdade de Enfermagem. A análise mostra ainda que esses trabalhos correspondem ao período de execução do PLE nos Estados, ou seja, entre 1989 e 1996.

A seguir, realizamos uma análise da produção apresentada acima segundo sua natureza e finalidade.

Almeida ${ }^{6}$, em estudo descritivo sobre a organização da categoria de enfermagem, do mercado de trabalho e das estratégias de regulação deste mercado, apropriou-se dos princípios da sociologia das profissões para discutir aspectos da profissionalização da enfermagem, apontando a qualificação do atendente de enfermagem como proposta para melhoria da qualidade da assistência. 0 autor caracterizou a participação majoritária do atendente na equipe de enfermagem como produto da divisão social do trabalho verificado na profissão e como consequência da lógica do rebaixamento de custo operacional dos serviços. Descreveu o Projeto Larga Escala como uma das opções de qualificação da categoria.

Azevedo ${ }^{7}$, por meio de estudo fenomenológico, investigou até que ponto a metodologia de base problematizadora priorizada pelo PLE foi implementada pela Escola de Auxiliar de Enfermagem do INAMPS no Rio de Janeiro, em seu curso de qualificação profissional. Utilizou como referencial o manual de capacitação pedagógica para os enfermeiros que atuaram como supervisores no projeto, bem como aportes teóricos nas linhas de educação crítica, teoria cognitiva e fenomenologia. Como resultado, constatou que a prática pedagógica utilizada aproximou-se em parte dos pressupostos do Larga Escala, principalmente na relação professor-aluno e na questão da problematização.

Torrez ${ }^{8}$, como executora do Projeto no Estado do Rio de Janeiro, procedeu a um estudo exploratório sobre a construção do PLE nesse Estado, por meio da fala dos diversos atores envolvidos (egressos, docentes e diretores entrevistados). A autora propõe a possibilidade de uma qualificação que aposta nos pressupostos teóricos metodológicos (integração ensinoserviço e metodologia problematizadora).

Oliveira ${ }^{9}$, na sua dissertação sobre a profissionalização dos trabalhadores de enfermagem nos serviços de saúde, buscou 
compreender o processo de construção da modalidade de qualificação profissional - PLE - na formação de auxiliares de enfermagem dos serviços de saúde de São Paulo. Foram sujeitos de análise 60 atendentes de enfermagem egressos de cinco cursos do PLE, sendo utilizada a perspectiva de compreensão da realidade de construção de um processo de formação no trabalho. Os resultados indicaram que o processo de qualificação propicia a formação global mediada pela percepção crítica da realidade. Contudo, conclui que a efetiva integração da dimensão da formação profissional no espaço de trabalho em saúde requer uma aproximação maior das políticas, visando à formação de recursos humanos.

Já Araújo ${ }^{10}$ analisou o processo de formação de duas turmas de auxiliares de enfermagem no período de 1993 a 1996, na perspectiva de uma reflexão sobre o processo educativo, objetivando identificar os marcos do currículo adotado, conteúdos e metodologia utilizada pelos egressos no cotidiano de seu trabalho. Como parâmetro, recorreu ao objetivo preconizado pelo PLE, isto é, melhorar a relação do alunotrabalhador com a realidade de seu ambiente. Investigou a prática dos auxiliares de enfermagem egressos de cursos do PLE, considerando o processo de formação a que foram submetidos, para verificar se ocorreram comportamentos distorcidos, inadequados ou equivocados nas suas práticas. Os resultados apontaram que as mudanças de atitudes foram pouco significativas, concluindo que não foi efetivada a assimilação esperada. As ações continuaram a ser executadas de maneira repetitiva e desarticulada.

Almeida ${ }^{11}$, em seu estudo qualitativo, faz a análise de como os princípios políticos do Sistema Único de Saúde foram incorporados aos cursos do Programa de Formação de Pessoal em Larga Escala - PLE. Entrevistas com os Coordenadores do PLE da Secretaria Municipal de Saúde de São Paulo, no período de 1989 a 1992, constituíram essa análise documental. Os resultados evidenciaram que a gestão municipal estava comprometida com a proposta de qualificação dos trabalhadores. Os coordenadores mostraram-se motivados para desenvolver o PLE, entretanto os coordenadores regionais mostraram uma compreensão limitada do processo de qualificação, separando, segundo a autora, a dimensão técnica da dimensão política do PLE.

Aguiar Neto ${ }^{12}$ manifestou sua preocupação com o processo de qualificação como prática social e partiu da concepção materialista histórica para analisar os atendentes de enfermagem formados como auxiliares pelo PLE na cidade de São Paulo, no período de 1990 a 1992. Utilizou como método de abordagem histórias de vida. Foram coletadas cinco histórias, nas quais buscou verificar se a qualificação promoveu mudanças na forma de pensar e agir no trabalho e na vida, perpassando pelo trabalho dos atendentes e pelas relações decorrentes da divisão social e técnica do trabalho. 0 resultado do trabalho permitiu reconhecer as possibilidades e os limites do PLE; entretanto, conforme conclusão da autora, não se constituiu de força capaz de suscitar transformaç̃̃es no modelo de atenção à saúde, o que denotou certa fragilidade na apreensão da dimensão política proposta pelo PLE para a qualificação de seus trabalhadores.

$\mathrm{Na}$ análise dos conteúdos da produção mencionada, observamos tendências a estudos relacionados aos atendentes de enfermagem, particularmente aos egressos dessa modalidade de formação, baseando-se na proposta de desenvolver uma integração entre educação e trabalho. Quatro dissertações exploraram esta temática objetivando verificar se a qualificação, através deste modelo, promoveu mudanças no trabalho e nas atitudes dos atendentes de enfermagem.

Como exemplo, citamos os estudos de Oliveira ${ }^{9}$ e Araújo ${ }^{10}$, o primeiro de caráter exploratório com abordagem hermenêutica-dialética e o segundo com enfoque dialético, ambos estruturados a partir das considerações metodológicas referendadas por Minayo ${ }^{13}$. Nesta mesma linha, a partir de uma concepção materialista histórica, Aguiar Neto ${ }^{12}$ utilizou como ferramenta metodológica para coleta das informações a história de vida para entender as mudanças ocorridas no cotidiano dos atendentes de enfermagem.

A metodologia utilizada por Torrez ${ }^{8}$ é exploratória, enfocando o momento da implantação do projeto no Rio de Janeiro, e adquiriu uma perspectiva mais abrangente, visto que estendeu seu quadro de análise aos outros atores envolvidos, como os diretores e docentes responsáveis pelo projeto.

Almeida ${ }^{11}$ empregou a análise documental e entrevistas realizadas com os coordenadores, gerentes e instrutores, segundo a técnica de análise de conteúdo. Por outro lado, 0 estudo de Azevedo lançou mão da fenomenologia, com o intuito de analisar a prática pedagógica do professor inserido nos cursos do PLE no Rio de Janeiro.

A partir desta aproximação inicial, foi possível constatar o predomínio de uma metodologia de investigação respaldada em dados qualitativos, exceto o estudo de Almeida ${ }^{6}$, que se configurou como estudo de reflexão teórico e se desenvolveu segundo uma perspectiva sociológica.

\section{$\checkmark$ Conjunto de artigos científicos}

Da produção de dez artigos científicos sobre o PLE, oito pertencem a periódicos da área de saúde, como a Revista Brasileira de Enfermagem, Revista Latino-Americana de Enfermagem e Revista da Escola de Enfermagem da USP, Cadernos de Saúde Pública e Educação Médica e Saúde; dois artigos publicados em livro sobre o Ensino Médio de Enfermagem e um artigo, mais recente, publicado em livro da Escola Politécnica de Saúde Joaquim Venâncio sobre Políticas de Saúde.

Em artigo de Santos, Souza e Galvão ${ }^{14}$ sobre a formulação de uma política de profissionalização para o pessoal de enfermagem sem qualificação específica, empregada no setor da saúde, os participantes da formulação do projeto sinalizam para uma política que valorize e garanta condições dignas de 
trabalho. Apresentam o PLE como uma política de profissionalização capaz de corrigir as distorções nos serviços de saúde e consideram o ambiente de trabalho como lócus da aprendizagem, visando ao não-afastamento do trabalhador do seu serviço.

Romano et al. ${ }^{15}$ trazem o contexto da implantação do PLE na Escola de Formação Técnica em Saúde "Enfermeira Isabel dos Santos". Introduziram o tema enfocando a ausência de investimentos na formação dos trabalhadores de saúde, contribuindo com dados do Inquérito Médico Sanitário e da pesquisa da força de trabalho em enfermagem. São descritos 0 histórico da escola, sua estrutura e recursos. No desenvolvimento do texto, apresentaram a concepção pedagógico-metodológica e a execução descentralizada dos cursos nos municípios.

Dal Poz et al. ${ }^{16}$, como executores do projeto, abordam o tema trazendo o histórico, estrutura e recursos para a execução do Projeto Larga Escala, oficializados pelo Instituto Nacional de Previdência e Assistência Social (INAMPS), a partir de 1986. Dentre os objetivos propostos, selecionam prioritariamente cursos de qualificação profissional via supletiva de ensino à clientela já engajada na força de trabalho, integração ensinoserviço, execução descentralizada dos cursos e concepção pedagógica e metodológica.

Santos e Souza ${ }^{4}$ apresentam texto similar ao publicado em 1988, pontuando a construção da experiência do PLE, apontando para a legitimação da força de trabalho em enfermagem e a formulação desse projeto para resolver 0 problema da qualificação.

Gryschek et al..$^{17}$ discorrem sobre um projeto de formação dos atendentes de enfermagem, o PLE, seu histórico, a legislação que o ampara e a sua trajetória na Secretaria de Saúde do Município de São Paulo.

Aguiar Neto e Soares ${ }^{18}$ reapresentam, como artigo, a síntese da dissertação de mestrado publicada em 2001, que buscava analisar as transformações ocorridas no trabalho, vida e maneira de pensar e agir dos atendentes de enfermagem que cursaram o PLE.

Amâncio Filho e Almeida ${ }^{19}$, em artigo sobre o ensino médio profissionalizante de enfermagem, discorrem sobre o ensino profissionalizante em saúde e comentam as possibilidades de formação para os trabalhadores inseridos no mercado de trabalho. Dentre elas, a estratégia pedagógica denominada Projeto Larga Escala, resultado de acordo institucional entre o Ministério da Educação, da Previdência e Assistência Social e Organização Pan-Americana da Saúde. Explicitam a organização desse movimento em todo o território nacional, nos Centros Formadores ou Escolas Técnicas de Saúde, e suas características, salientando que o aluno deve ser considerado como sujeito do processo ensino-aprendizagem, com a adoção de um sistema permanente de avaliação, e relacionando os docentes como os próprios profissionais em serviço.

Bento e Bueno ${ }^{20}$ tecem comentários sobre os propósitos, objetivos, funcionamento, grade curricular, carga horária e aspectos educacionais que envolvem os projetos Larga Escala e
Classe Descentralizada. Estes projetos, embora ocorrendo concomitantemente, divergem da proposta de formação. O PLE apresenta como proposta o currículo integrado; os professores não são remunerados (são os próprios enfermeiros da unidade) e o curso de longa duração, com estágio e aulas nos serviços, é restrito à rede pública.

Guimarães ${ }^{21}$ relata sua experiência na participação no PLE no município de Campinas/SP, no período entre dezembro de 1988 e dezembro de 1996. Descreve a experiência pedagógica e seus aspectos relevantes, como a utilização da pedagogia da problematização; o currículo integrado, com momentos de comunicação e dispersão; e a organização do currículo, privilegiando, além das disciplinas profissionalizantes; tópicos referentes à cidadania, profissão e trabalho em saúde.

Pereira ${ }^{22}$ resgata as conferências nacionais de saúde, correlacionando a organização do sistema de saúde com os projetos voltados para a formação profissional dos trabalhadores inseridos neste sistema. Discute a Reforma Sanitária e o Projeto de Formação em Larga Escala, como concepção de uma prática educativa, voltada para os trabalhadores, que incorporava a reflexão e a percepção, construindo a capacidade de pensar e planejar ações de saúde.

Os artigos sobre a questão foram produzidos entre $1988 \mathrm{e}$ 2005 e se debruçam sobre a descrição do projeto, propõem registrar e divulgar uma experiência pedagógica. Os quatro primeiros veiculam as propostas do PLE, e seus autores, Santos, Souza e Galvão, são os responsáveis pela formulação e execução do PLE em nível federal. Já Romano ${ }^{15}$ e Dal Poz et al. ${ }^{16}$ são do gênero relato de experiência e trazem a execução do PLE no Estado do Rio de Janeiro, bem como Gryschek ${ }^{17}$ e Guimarães ${ }^{21}$, nos municípios de São Paulo e Campinas, respectivamente. Aguiar Neto ${ }^{18}$ reapresenta, como artigo, a síntese de sua dissertação de mestrado. Pereira ${ }^{22}$ procura resgatar o PLE inserido em um momento político que favorecia a sua institucionalização.

Em linhas gerais, a análise dos artigos científicos dá indicadores de uma produção baseada em trabalhos do gênero relato de experiência e de análise teórica, em detrimento de outros gêneros de pesquisa.

\section{$\checkmark$ Conjunto de boletins informativos}

Outros materiais encontrados durante a revisão de literatura, de aspecto mais restrito, foram os boletins - formas de difusão de conhecimento para as diversas instituições de saúde pública. Os boletins informativos envolvem publicações de caráter oficial e informal e veiculavam informações sobre a concepção do projeto. Catalogamos três boletins, sendo que um deles é fonte oficial do Ministério da Saúde e dois são documentos mimeografados e confeccionados pelas equipes das regionais de saúde.

O Boletim Informativo Recursos Humanos para o Sistema Único de Saúde - RH SUS ${ }^{23}$ discute a experiência da Escola de Formação Técnica em Saúde "Izabel dos Santos", que pretendia 
qualificar nove mil atendentes de enfermagem. 0 documento divulga a experiência, assim como apresenta os princípios pedagógicos e metodológicos do projeto.

0 Boletim Informativo do Escritório Regional de Saúde ERSA 2 - Butantã ${ }^{24}$ registra a formação da primeira turma de auxiliares de enfermagem pelo PLE e as dificuldades e desafios postos neste tipo de inovação, como ausência de material didático, pouco envolvimento das unidades básicas de saúde no projeto e o tempo de duração do curso, que foi alvo da maioria das críticas.

O Boletim do Sistema Unificado e Descentralizado de Saúde - SUDS R -Ribeirão Preto ${ }^{25}$ discute a situação do projeto no Estado de São Paulo (Ribeirão Preto e região) informando que os sujeitos participantes das atividades do PLE tiveram o benefício da liberação de ponto. Em contrapartida, houve sobrecarga de trabalho, falta de ajuda de custo, já que havia a necessidade de viajar, desconhecimento e incompreensão de colegas, o que exigiu um grande esforço para a viabilização do projeto.

0 último estudo inventariado e que não se insere nos conjuntos apresentados acima foi realizado por Germano ${ }^{26}$, que objetivou delinear a História do Centro de Formação de Pessoal para os Serviços de Saúde (CEFOPE), Rio Grande do Norte, desde sua criação em 1984 até 1996. Neste período, o CEFOPE utilizou, para formação de pessoal de nível médio da área de saúde, a proposta de Formação em Larga Escala e colocou-se entre as experiências pioneiras nesta modalidade de formação. A autora registrou a experiência do PLE como modelo metodológico utilizado pela instituição.

\section{CONSIDERAÇÕES FINAIS}

Este trabalho permitiu mapear e discorrer sobre o que foi publicado acerca do PLE. Faz-se necessário iniciar uma nova tarefa, que é o aprofundamento da leitura deste material, o que significa uma análise crítica do que foi produzido, tanto no seu aspecto teórico-epistemológico quanto no político-ideológico.

Além disso, o estudo de Oliveira ${ }^{9}$ nos diz que, apesar de o PLE ter gerado discussões e polêmicas, foi pouco avaliado enquanto processo de qualificação profissional. Segundo a autora, existiam muitos significados a serem explorados e desvendados.

Nesse sentido, podemos ver, com essa primeira aproximação, o pequeno número de investigações realizadas e o desconhecimento dos resultados do Projeto Larga Escala em âmbitos locais e regionais. Desta forma, este estudo nos permitiu verificar que o PLE foi pouco investigado, considerando que foi um projeto desenvolvido em todo o país durante um período de tempo relativamente longo.

No entanto, os estudos aqui apresentados contribuíram para apontar caminhos e revelar silêncios e lacunas existentes sobre este projeto de formação, como uma proposta mais abrangente que envolva a avaliação desse processo como um todo.
Isso reforça a importância de dar continuidade a este trabalho, pois observamos um número significativo de estudos mobilizados pela concepção pedagógica e metodológica, e não pela compreensão histórica desta experiência, que é uma das intenções de nossa pesquisa, em que vislumbramos a possibilidade de preencher algumas lacunas do conhecimento existentes na história da enfermagem brasileira.

\section{REFERÊNCIAS}

1.Santos NMP. A produção de conhecimento: do ensino à prática de enfermagem. Esc Anna Nery Rev Enferm [on-line] 2002 ago [citado 15 maio 2008] 6(2): 169-74. Disponível em: http://www.eean.ufr..br/ revista_enf/revistas.htm.

2.Ministério da Saúde (BR). Recursos humanos para serviços básicos de saúde: formação de pessoal de níveis médio e elementar pelas instituições de saúde. Brasília (DF); 1982.

3.Lei $n^{0} 5.692$ de 11 de agosto de 1971. Diretrizes e Bases para o ensino de $1^{\circ}$ e $2^{\circ}$. Brasília (DF); 1971.

4.Santos I, Souza AA. Formação de pessoal de nível médio pelas instituições de Saúde: Projeto Larga Escala, uma experiência em construção. Saude Debate 1989 mar; 24: 61- 4.

5.Ferreira NSA. As pesquisas denominadas "estado da arte". Cad CEDES 2002 ago; 23(79): 257-72.

6.Almeida JLT. A qualificação do atendente no processo de profissionalização de enfermagem. [dissertação de mestrado] Rio de Janeiro (RJ): Instituto de Medicina Social/ UERJ; 1992.

7.Azevedo ML. Educação de trabalhadores da enfermagem com enfoque na pedagogia da problematização: avaliação de uma experiência no Rio de Janeiro [dissertação de mestrado] Rio de Janeiro (RJ): Faculdade de Educação/ UFRJ; 1992.

8.Torrez MNFB. Qualificação e trabalho em saúde: o desafio de "ir além" na formação dos trabalhadores de nível médio. [dissertação de mestrado] Rio de Janeiro (RJ): Faculdade de Educação/ UFRJ; 1994.

9.Oliveira LSS. A profissionalização dos trabalhadores de enfermagem nos serviços de saúde: uma experiência de construção do Projeto Larga Escala na cidade de São Paulo. [dissertação de mestrado] São Paulo (SP): Faculdade de Saúde Pública/USP; 1996.

10Araújo MCF. Educação e profissionalização do auxiliar de enfermagem: uma prática em discussão. [disser tação de mestrado] Bahia (BA): Faculdade de Educação/UFBA; 1999.

11.Almeida AH. A incorporação dos princípios e das diretrizes do Sistema Único de Saúde aos cursos de formação do auxiliar de enfermagem: o Projeto Larga Escala no período de 1989 a 1992. [dissertação de mestrado] São Paulo (SP): Faculdade de Saúde Pública/USP; 2000.

12.Aguiar Neto Z. A qualificação dos atendentes de enfermagem: transformação no trabalho e na vida. [dissertação de mestrado] São Paulo (SP): Faculdade de Enfermagem/USP; 2001.

13.Minayo MCS. 0 desafio do conhecimento: pesquisa qualitativa em saúde. $4^{a}$ ed. São Paulo (SP): Hucitec; 1996.

14.Santos I, Souza AMA, Galvão EA. Subsídios para a formulação de uma política de profissionalização para o pessoal de enfermagem, 
sem qualificação específica, empregada no setor saúde - Brasil. Educ Med Salud. 1988; 22 (2): 200 - 10.

15.Romano RAT, Torrez MNFB, Cortez MV, Mendes RN. A experiência de implantação da Escola de Formação Técnica em Saúde "Enfa Izabel dos Santos" pela Secretaria de Estado de Saúde no Rio de Janeiro. Rev Bras Enferm 1991 out-dez; 44(4): 7-10.

16.Dal Poz MR, Romano RAT, Torrez MNFB, Cortez MV. Formação de recursos humanos de nível médio em saúde no Rio de Janeiro: a experiência da Escola Técnica em Saúde Enfermeira Izabel dos Santos. Cad Saude Publica 1992 jan/mar; 8 (1): 57 - 61.

17.Gryschek ALFP, Almeida AH, Antunes MN, Miyashiro SY. Projeto Larga Escala: uma proposta pedagógica atual. Rev Esc Enferm USP. 2000 jun; 34 (2): 196-201.

18.Aguiar Neto Z, Soares CB. A qualificação dos atendentes de enfermagem: transformações no trabalho e na vida. Rev Latino - am Enfermagem 2004 jul/ago; 12(4): 614-22.

19.Amâncio Filho A, Almeida JLT. Ensino profissionalizante em saúde. Boletim Técnico do SENAC [on-line] jan/abr 1995; [citado 06 ago 2005]; 21 (1): [aprox 9 telas]. Disponível em www.senac.br/ informativo/bts/211/2101038045.pdf.

20.Bento ICB, Bueno SMV. Contribuição para o estudo da aplicabilidade técnica dos Projetos Larga Escala e Classe Descentralizada destinados à formação de auxiliares de enfermagem. In: Bueno et al, organizadoras. Enfermeiro-professor e o ensino médio em enfermagem. Ribeirão Preto (SP): Fundação Instituto de Enfermagem de Ribeirão Preto; 1998.

21.Guimarães AL. Formação de auxiliar de enfermagem através do PLE: a experiência do município de Campinas/ SP. In: Bueno et al, organizadoras. Enfermeiro-professor e o ensino médio em enfermagem. Ribeirão Preto (SP): Fundação Instituto de Enfermagem de Ribeirão Preto; 1998.

22.Pereira IB. Políticas de saúde e formação do trabalhador. In: Pereira IB,organizador. Políticas de Saúde. Rio de Janeiro(RJ): Fiocruz; 2005. p.117-42.

23.Ministério da Saúde (BR). Boletim Informativo. Recursos Humanos para o SUS - RH SUS. Brasília (DF); 1992.

24.Projeto Larga Escala. Boletim Informativo ERSA 2 Butantã. 1992; 3 (5).

25.Projeto Larga Escala. Boletim Informativo SUDS Ribeirão Preto. $1^{\text {a }}$ ed. 1988 set; 1. Manuscrito.

26.Germano RM. CEFOPE/RN: uma experiência de formação de recursos humanos em saúde 1984-1996. Natal (RN): SSAP; 1996. 